\title{
El timerosal en la práctica pediátrica
}

\author{
LM. Fernández Cuesta \\ Pediatra. CS de Grado. Grado, Asturias. España.
}

Fecha de publicación en Internet: 12 de noviembre de 2010

\begin{abstract}
Resumen
El mercurio no tiene ninguna función fisiológica en el cuerpo humano y está ampliamente distribuido en la naturaleza. Puede ser tóxico por inhalación, ingestión o contacto. El timerosal es una sal orgánica de mercurio usada como antiséptico y antifúngico desde 1928. Desde finales de la década de 1990, el mercurio empezó a ser retirado de los medicamentos y materiales de uso clínico. Se ha hecho un esfuerzo para eliminar el timerosal de las vacunas siguiendo el principio de precaución, pues no hay evidencias científicas que avalen un daño cerebral atribuible al timerosal. No se ha encontrado ninguna asociación entre su uso y el riesgo de desarrollar autismo. Actualmente, en los países de nuestro entorno solo se usa en muy pocas vacunas en envases multidosis, y todas las incluidas en los calendarios oficiales españoles, así como las de uso común en niños fuera de ellos (neumocócicas y rotavirus), están libres de timerosal. No obstante, la OMS ha reiterado que las vacunas que contienen timerosal pueden seguir utilizándose, especialmente en el Tercer Mundo donde, por necesidades logísticas, se utilizan envases multidosis, pues el riesgo real de enfermedad y muerte por enfermedades vacunables en quienes no se vacunan es muy superior al riesgo hipotético derivado del su uso. Como con cualquier otro medicamento, pueden presentarse reacciones de hipersensibilidad al timerosal, generalmente locales. Los esfuerzos investigadores sobre determinados problemas neurológicos como el autismo deberían encaminarse a buscar sus verdaderas causas en lugar de a sembrar dudas sobre la seguridad de las vacunas.
\end{abstract}

Palabras clave: Mercurio. Toxicidad. Vacunas.

\section{Thimerosal in pediatric practice}

\section{Abstract}

Mercury has no physiological function in the human body and is widely distributed in nature. It may be toxic by inhalation, ingestion or contact. Thimerosal is an organic salt of mercury used as an antiseptic and antifungal since 1928. Since the late 1990s, mercury began to be withdrawn from drugs and materials for clinical use. An effort has been made to remove thimerosal from vaccines as a precautionary principle, since there is no scientific evidence to substantiate that brain damage can be attributable to thimerosal. No connection has been found between its use and the risk of developing autism. In the Western World it is only currently used in very few vaccines in multidose containers, whereas all those included in Spa-

Luis M. Fernández Cuesta, drlmfcuesta@wanadoo.es

El autor declara no presentar conflictos de intereses en relación con la preparación y publicación de este artículo. 
nish Schedules, as well as in those commonly used for children outside them (pneumococcal and rotavirus), are free from thimerosal. However, the WHO has reiterated that vaccines containing thimerosal may still be used, especially in the Third World where, for logistical needs, multidose containers are used, as the real risk of illness and death by vaccianble diseases in those who do not get vaccinated is much higher than the hypothetical risk arising from their use. As with any medicine, there may be hypersensitivity reactions to thimerosal, usually local. Research efforts should be directed towards finding the real causes of neurological problems like autism instead of throwing doubts about the safety of vaccines.

Key words: Mercury. Toxicity. Vaccine.

\section{El mercurio}

El mercurio es un elemento químico que se encuentra en la naturaleza de tres formas: como metal, formando sales inorgánicas (sulfuros y otras) y como sales orgánicas (etil-, metil- y fenilmercurio). Su distribución es muy amplia, por lo que no es posible evitar totalmente la exposición. Los peces grandes depredadores (atún) y algunos crustáceos son las principales fuentes de mercurio orgánico de la dieta (en forma de metilmercurio), especialmente si viven en aguas contaminadas. En las poblaciones que no están expuestas por motivos laborales, los alimentos son la fuente principal de mercurio'. La ingesta alimentaria promedio de mercurio varía en los diversos países entre 2 y $20 \mathrm{~g} /$ día por persona². También estamos expuestos en menor medida a través del ambiente y de algunos productos del hogar (plaguicidas, pilas, pinturas antisuciedad), algunos medicamentos y las amalgamas dentales.

\section{Efectos del mercurio en el organismo}

El mercurio no tiene ninguna función fisiológica en el cuerpo humano. En cualquiera de las formas en que se presenta puede ser tóxico, aunque de distinta manera ${ }^{3}$. La toxicidad depende de la forma química, la vía de entrada, la dosis, la duración de la exposición y la edad. Es dañino por inhalación, ingestión y contacto. Por ejemplo, la tasa de absorción gastrointestinal para el mercurio elemental es del 0,01\%, mientras que por vía inhalada es del $80 \%$. En cambio, la tasa de absorción por ingestión del mercurio orgánico (metilmercurio), forma en la que se encuentra en peces contaminados, llega a ser del $90 \%$ al $100 \%$.

Los efectos inmediatos que puede producir la inhalación aguda de vapores de mercurio son: escozor de garganta, dolor de cabeza, náuseas, pérdida del apetito y debilidad muscular.

En la mayoría de los casos, tras tocar mercurio es suficiente con lavarse la zona 
con agua y jabón, aunque en contactos prolongados puede provocar enrojecimiento en los ojos e irritación de la piel.

En caso de ingestión de pequeñas cantidades, basta con enjuagar la boca con agua. En grandes cantidades se debe practicar lavado gástrico, catarsis o emesis y utilizar quelantes (el ácido 2,3dimercaptosuccínico parece ser el más eficaz, aunque la experiencia en niños es muy limitada) ${ }^{4}$.

Exposiciones prolongadas o a elevadas cantidades de cualquier forma química pueden dañar el sistema nervioso, el riñón y al feto, provocando lo que conocemos como hidrargirismo, patología observada desde antiguo entre los trabajadores del mercurio (mineros, sombrereros, etc.).

La ingestión prolongada de alimentos contaminados con mercurio provoca la enfermedad conocida como de Minamata, que debe su nombre a la bahía japonesa donde se describió por primera vez en 1956. Se trata de un claro ejemplo de cómo un agente tóxico puede transmitirse a través de la cadena trófica hasta llegar al ser humano. Cientos de pescadores y sus familias fueron gravemente intoxicados por metilmercurio, el cual se había bioacumulado en los peces como resultado de la liberación en la bahía de los desechos de mercurio de una planta química. Los efectos neurológicos se observaron tanto en niños como en adultos (parestesia, temblores, alteraciones sensoriales y de la audición). Sin embargo, la manifestación más dramática fue la enfermedad adquirida intrauterinamente caracterizada por parálisis cerebral, ceguera y retraso mental, que se presentó en los nacidos de madres con altas concentraciones de mercurio. Otros incidentes similares ocurrieron en Alamogordo (Nuevo México) y en Iraq, donde la fuente de exposición fue el consumo de trigo tratado con fungicidas a base de mercurio.

Los efectos tóxicos de los compuestos orgánicos como el metilmercurio (el más estudiado) son más selectivos para el sistema nervioso, y es posible que también ocurra así con el fenil- y el etilmercurio. Además, se absorben con facilidad por la piel, por vía digestiva y por inhalación, y luego se distribuyen ampliamente por todo el organismo. El metilmercurio puede pasar la barrera hematoencefálica y concentrarse en el cerebro, y pasar la barrera placentaria y llegar al feto. En el feto puede producir daño cerebral, retraso mental, ceguera, falta de coordinación, convulsiones e incapacidad para hablar ${ }^{5-7}$. También los niños, por su menor peso y porque su sistema nervioso central está en desa- 
rrollo (especialmente en los menores de dos años), pueden sufrir daño cerebral que cause irritabilidad, temblores, alteraciones de la vista o del oído. Hay estudios que sugieren que grandes exposiciones intermitentes producen más daño neuropsicológico en niños que las pequeñas pero mantenidas. No está claro si con el etilmercurio pasa lo mismo. Los estudios farmacocinéticos sugieren que la vida media del etilmercurio es significativamente más corta en comparación con el metilmercurio8.

Por analogía, dada su similitud quími$\mathrm{ca}$, se tiende a asumir con reservas para el timerosal las recomendaciones hechas por los principales organismos internacionales (OMS, EPA, FDA) con respecto al metilmercurio. Sin embargo, tampoco hay unanimidad en el punto de corte $y$, siempre en referencia a exposiciones prolongadas, los límites de seguridad se sitúan entre los 0,1 y los $3,3 \mu \mathrm{g} / \mathrm{kg}$ de peso y día. Se estima que para niños y embarazadas estos límites podrían ser menores.

\section{Timerosal}

El timerosal, también llamado tiomersal o mertiolate, es una sal orgánica de mercurio cuyo nombre químico es tiosalicilato de etilmercurio sódico $\left(\mathrm{C}_{9} \mathrm{H}_{9} \mathrm{HgNaO}{ }_{2} \mathrm{~S}\right)$, (aproximadamente un $49 \%$ de mercurio en peso), que se usa como antiséptico y agente antifúngico. En el cuerpo, se metaboliza a catión tiosalicilato y etilmercurio $\left(\mathrm{C}_{2} \mathrm{H}_{5} \mathrm{Hg}+\right)$, que es el responsable de su acción antimicrobiana.

Fue desarrollado y registrado bajo el nombre comercial de Merthiolate ${ }^{\circledast}$ o Mertodol $^{\circledR}$ en 1928 por la corporación farmacéutica Eli Lilly and Company ${ }^{\circledR}$ y desde entonces se ha usado como un preservante en vacunas, preparaciones de inmunoglobulinas, antígenos para diagnóstico de alergias, antisueros, medicamentos tópicos nasales y oftálmicos y tintes de tatuajes.

En su día, el timerosal evitó efectos adversos serios tales como la infección por Staphylococcus que, en un incidente en 1928, produjo la muerte a 12 de 21 niños inoculados con una vacuna de difteria que carecía de preservante ${ }^{9}$. A diferencia de otros preservantes de vacunas usados por entonces, el timerosal no reducía la potencia de las vacunas ${ }^{10}$.

Desde que en 1997 la Food and Drug Administration norteamericana comenzase a revisar y evaluar el riesgo de los alimentos y medicamentos que contenían mercurio y en 1999 lanzase una serie de medidas cautelares junto con la American Academy of Pediatrics ${ }^{11,12}$, se ha hecho un gran esfuerzo para eliminar el timerosal de las vacunas, no tanto 
porque se constatase fehacientemente la materialización del riesgo, sino más bien por el principio de precaución, pues no existen en la actualidad evidencias científicas que avalen un daño cerebral atribuible al timerosal.

En Estados Unidos, la Unión Europea y otros países se está retirando este compuesto de las vacunas que se aplican rutinariamente a los niños. La distribución de vacunas en ampollas individuales elimina la necesidad de un agente bacteriostático como el timerosal. En la actualidad, en los países de nuestro entorno solo se usa en muy pocas vacunas en envases multidosis.

Además, el mercurio ya ha sido retirado de la mayoría de los medicamentos, así como de materiales de uso clínico como termómetros o esfingomanómetros $^{13}$.

Se han llevado a cabo pocos estudios de la toxicidad de timerosal en seres humanos. Los experimentos en animales sugieren que se disocia rápidamente para liberar catión etilmercurio después de la inyección, que los patrones de disposición del mercurio son similares a los de haber sido expuesto a dosis equivalentes de cloruro de etilmercurio, y que el sistema nervioso central y los riñones son los órganos diana. Se han observado signos similares en envenenamientos accidentales en seres humanos. Los mecanismos de acción del tóxico son desconocidos. La excreción fecal representa la mayor parte de la eliminación del cuerpo. El etilmercurio se elimina de la sangre con una vida media de aproximadamente 18 días, y del cerebro en aproximadamente 14 días. El mercurio inorgánico procedente de la metabolización del etilmercurio tiene un tiempo de eliminación mucho más largo, de al menos 120 días, aunque parece ser mucho menos tóxico que el mercurio inorgánico, por razones aún no comprendidas ${ }^{14}$.

\section{Vacunas}

Hasta hace unos años, algunas de las vacunas de uso común en España contenían timerosal como conservante, o trazas del mismo como restos del proceso de fabricación. Como se dijo antes, a finales de la década de los 90 surgieron dudas en Estados Unidos acerca de la inocuidad del timerosal. La causa fue la observación de que la cantidad acumulada de mercurio durante el plan de vacunación del lactante podía exceder el umbral recomendado de exposición al metilmercurio establecido por la Agencia de Protección del Medio Ambiente (Enviromental Protection Agency [EPA]). A este último compuesto se le achacaba la 
causa de anomalías neurológicas en el recién nacido por haber estado expuesto durante el periodo fetal a las altas dosis ingeridas por la madre durante un largo periodo. Como resultado de esta preocupación basada en datos teóricos, en 1999 los organismos consultivos estadounidenses encargados de la inmunización recomendaron acelerar la eliminación del timerosal de las vacunas ${ }^{11}$. Esta decisión movió a otros países a dar el mismo paso. Tiempo después se demostró que las características farmacocinéticas del etilmercurio son muy distintas a las del metilmercurio y que aquel se excreta rápidamente por vía intestinal ${ }^{15}$.

Nadie ha podido demostrar hasta la fecha que el timerosal incluido en las vacunas interfiera en el desarrollo cerebral del feto o el lactante, ni que produzca trastornos del desarrollo psicomotor del niño ${ }^{16,17}$, por lo que, al hablar de riesgos, tenemos que hacerlo desde un punto de vista meramente especulativo.

Según los calendarios vacunales españoles anteriores a 2004, la exposición a etilmercurio por las vacunas que contienen timerosal durante los seis primeros meses de vida variaba desde 0 hasta $187 \mu \mathrm{g}$, dependiendo de las marcas comerciales utilizadas. La mayoría de los niños recibieron poco o nada de etil- mercurio, pero en el caso de la peor opción (ante la posibilidad de optar entre varias marcas, recibir siempre la vacuna que contiene timerosal) solo excepcionalmente se habrían superado, al completar el calendario, las recomendaciones más rigurosas de la EPA para el metilmercurio. Para que, en una sola visita, la exposición aguda hubiera superado la dosis tóxica, habría sido necesario que se tratase de un gran prematuro de muy bajo peso que recibiese a la vez al menos dos vacunas con timerosal. Hasta ese momento, aplicando las recomendaciones del Consejo Interterritorial del Sistema Nacional de Salud para el calendario vacunal español de 2004, las dosis máximas de mercurio que podría haber recibido un niño antes de los 14 años serían insignificantes (el contenido en las vacunas de tétanos-difteria tipo adulto y las trazas contenidas en la vacuna de hepatitis $B$, en caso de utilizar una determinada marca comercial).

Dos grupos de expertos independientes, el Comité Consultivo Mundial sobre Inocuidad de las Vacunas (GACVS), creado por la OMS a tal efecto ${ }^{18}$, y el Instituto de Medicina de los Estados Unidos (Institute of Medicine of the Nacional Academies [IOM]), han seguido revisando la cuestión y no han encontrado datos científicos que permitan 
probar la toxicidad de las vacunas que contienen timerosal. En consecuencia, el Grupo Consultivo Estratégico de Expertos de la OMS ha afirmado reiteradamente y de modo terminante que las vacunas que contienen timerosal pueden seguir utilizándose para mantener la seguridad de la inmunización, especialmente en el Tercer Mundo, donde las necesidades logísticas ameritan la utilización de envases multidosis. Para estos casos, dado que la punción repetida de la cubierta de goma del envase y la extracción de la vacuna entraña un riesgo de contaminación, no existe ningún otro conservante con un perfil de seguridad y eficacia como el timerosal, que no puede suprimirse sin poner en peligro la calidad de las vacunas infantiles utilizadas en los programas mundiales ${ }^{19}$.

Actualmente, todas las vacunas incluidas en los calendarios oficiales de España, así como las de uso común en niños fuera de ellos (neumocócicas conjugadas y rotavirus), están libres de timerosal. Como dato anecdótico, la vacuna frente a la gripe pandémica H1N1, Pandemrix ${ }^{\circledast}$, contiene $0,005 \mathrm{mg}$ de timerosal por dosis de $0,5 \mathrm{ml}$, cantidad muy por debajo de cualquiera de los límites de seguridad marcados por las principales autoridades sanitarias para cualquier compuesto mercurial. La neumocócica polisacarídica y la de la gripe, indicadas en determinados niños con patologías crónicas, tienen también alternativas libres de mercurio.

En cualquier caso, no se puede olvidar, como se ha recordado desde la OMS, que el riesgo real de enfermedad y muerte por enfermedades vacunables en aquellos niños que no se vacunan es muy superior al riesgo hipotético derivado del uso del timerosal' ${ }^{19}$.

\section{Autismo}

Merece especial atención la asociación sugerida por algunos entre las vacunas infantiles y el autismo, pues ha sido una de las controversias más polémicas respecto a la seguridad vacunal en los últimos años. A pesar de la evidencia científica en contra de una asociación causal, muchos padres y movimientos antivacunas o autodenominados de defensa de los pacientes continúan difundiendo la sospecha de que las vacunas, especialmente la triple vírica (sarampión-parotiditis-rubéola) y las que contienen timerosal, son causa del autismo. Las pruebas científicas hasta la fecha no avalan esa causalidad ni para la una ni para las otras ${ }^{20}$.

Para rebatir estos argumentos, se han publicado numerosos trabajos que evaluaron la relación entre el timerosal y el 
autismo. Uno de ellos en particular tiene las dimensiones y la calidad suficientes como para responder a la cuestión con autoridad. El estudio de cohortes de base poblacional se realizó en Dinamarca ${ }^{21}$ sobre una amplia cohorte de niños nacidos entre 1990 y 1996. Se relacionó la información sobre las vacunaciones con el diagnóstico de autismo y otros síndromes del espectro autista y con potenciales factores de confusión. Se estableció el diagnóstico de autismo basándose en criterios estrictos. Se registraron todos los diagnósticos hasta el final del año 2000. Desde 1970, la única vacuna que contenía timerosal usada en Dinamarca era la antitosferina de células enteras. Esta vacuna se usó hasta marzo de 1992, cuando fue reformulada sin timerosal para seguir usándose hasta enero de 1997. La vacuna se administró a las cinco y a las nueve semanas, y a los diez meses, sin tener en cuenta su contenido en timerosal, equivalente a $25 \mu \mathrm{g}$ de etilmercurio la primera dosis y el doble las sucesivas, alcanzando una dosis máxima de $125 \mu \mathrm{g}$ en cada niño.

Se estudiaron 467450 niños con un seguimiento de prácticamente tres millones de personas al año. Hubo 440 casos de autismo y 878 casos de otros síndromes del espectro autista. Se perdió la información de 5770 niños (1,2\%), principalmente debido a la inmigración. De la cohorte, el 95,6\% recibieron al menos una dosis, el $89 \%$ dos dosis y el $63 \%$ las tres dosis de la vacuna. Solo el $4,4 \%$ no recibió ninguna dosis.

No se detectó asociación entre el uso de timerosal y el riesgo de desarrollar autismo o síndromes del espectro autista, ni se detectó un aumento del número de casos diagnosticados de autismo asociado al uso de la vacuna con timerosal. Tampoco se detectó relación dosis-respuesta con la exposición al etilmercurio. Los riesgos relativos se ajustaron en función de posibles factores de confusión, pero las tasas fueron incluso inferiores en los niños expuestos al etilmercurio.

Posteriormente, se han añadido nuevos estudios que se revisaron en un reciente metaanálisis. Se identificaron 12 publicaciones que cumplían los criterios de selección mediante una búsqueda bibliográfica: diez estudios epidemiológicos y dos estudios farmacocinéticos de etilmercurio. La mayoría de los trabajos epidemiológicos no apoyaban una asociación entre las vacunas con timerosal y el trastorno del espectro autista. Los estudios epidemiológicos que sí apoyaban la asociación eran de mala calidad y con defectos de diseño significativos que invalidaban sus conclusiones. Los estudios farmacocinéticos mostraron que la vida 
media del etilmercurio es significativamente más corta en comparación con el metilmercurio. Los estudios no demuestran un vínculo entre las vacunas que contienen timerosal y los trastornos del espectro autista, y la farmacocinética del etilmercurio hace aún menos probable tal asociación. Los revisores concluyen que las pruebas no apoyan un cambio en la práctica con respecto a la administración de vacunas que contienen timerosal en las zonas del mundo en las que se siguen utilizando8.

\section{Hipersensibilidad}

Por último, cabe mencionar que, al igual que con cualquier otro medicamento, pueden presentarse reacciones de hipersensibilidad al timerosal. Sin embargo, las cantidades contenidas en las vacunas no han sido suficientes para desencadenar reacciones adversas graves, ni siquiera en personas con sensibilización demostrada por patch o prick test. Más bien suelen ser reacciones locales de hipersensibilidad retardada (alrededor del $1 \%$ ), algunas persistentes, $y$ también urticarias y exantemas con menor frecuencia ${ }^{22}$. Lo único que cabría recomendar es cautela en aquellos pacientes de quienes se sepa que son alérgicos a los mercuriales, y sopesar la relación riesgo-beneficio en cada caso.

\section{Conclusión}

Como conclusión podemos decir que, aunque el mercurio es indudablemente tóxico y se han formulado múltiples hipótesis sobre la toxicidad neurológica de las vacunas que contienen timerosal, no ha podido demostrarse ninguna. Además, la cantidad contenida actualmente en algunas vacunas (que no se usan en España) es insuficiente para que esa supuesta toxicidad se materialice. Los esfuerzos investigadores sobre determinados problemas neurológicos o neuropsicológicos como el autismo deberán encaminarse a buscar sus verdaderas causas en lugar de a sembrar dudas sobre la seguridad de las vacunas.

Por otro lado, difícilmente nos atañe este problema cuando llevamos años utilizando vacunas libres de timerosal en los calendarios infantiles. Ya que disponemos de ellas, es preferible utilizarlas, especialmente en lactantes, por un principio de prudencia y por evitar alarmas sociales, aunque no hay motivo para que se rechacen las vacunas que lo contengan cuando se estimen necesarias, pues el beneficio de protección frente a la enfermedad supera con creces el riesgo potencial. Cabe volver a recordar la utilidad de las presentaciones multidosis en las campañas de vacunación en los 
países no desarrollados, y la necesidad de que la industria persevere en sus esfuerzos para encontrar alternativas al ti- merosal como conservante, con iguales o mejores perfiles tanto de seguridad como de eficacia.

\section{Bibliografía}

1. Mozaffarian D, RimmEB. Fish Intake, contaminants, and human health: evaluating the risks and the benefits. JAMA. 2006;296:1885-99.

2. Hojas de información sobre sustancias químicas. Mercurio. En: Guías para la calidad del agua potable. Primer apéndice a la tercera edición, volumen 1; Recomendaciones. Ginebra: Organización Mundial de la Salud; 2006. p. 318-9. Disponible en www.who.int/water_sanitation_health/dwq/gdw q3rev/es/index.html [consultado el 01/07/2010].

3. Clarkson TW. The toxicology of mercury. Crit Rev Clin Lab Sci. 1997;34:369-403.

4. Mahajan PV. Intoxicación por metales pesados. En: Kliegman RM, Behrman RE, Jenson $H B$, Stanton BF. Nelson Tratado de Pediatría. 18. a edición. Barcelona: Elsevier Saunders; 2009. p. 290912.

5. Cox C, Breazna A, Davidson PW, Myers GJ, Clarkson TW. Prenatal and postnatal methylmercury exposure and neurodevelopmental outcomes. JAMA. 1999.282:1333-4.

6. Davidson PW, Myers GJ, Cox C, Axtell C, Shamlaye C, Sloane-Reeves J, et al. Effects of prenatal and postnatal methylmercury exposure from fish consumption on neurodevelopment: outcomes at 66 months of age in the Seychelles Child Development Study. JAMA. 1998;280: 701-7.

7. Weil M, Bressler J, Parsons P, Bolla K. Blood mercury levels and neurobehavioral function. JAMA. 2005;293:1875-82.
8. Parker SK, Schwartz B, Todd J, Pickering LK. Thimerosal-containing vaccines and autistic spectrum disorder: A critical review of published original data. Pediatrics. 2004;114:793-804.

9. Center for Biologics Evaluation and Research, U.S. Food and Drug Administration. Thimerosal in Vaccines. Disponible en www.fda.gov/BiologicsBloodVaccines/SafetyAvailability/VaccineSafety/U CM096228 [actualizado el 03/31/2010; consultado el 26/06/2010].

10. Baker JP. Mercury, vaccines, and autism: one controversy, three histories. Am J Public Health. 2008;98:244-53.

11. American Academy of Pediatrics, Committee on Infectious Diseases and Committee on Environmental Health. Thimerosal in vaccines - An intern Report on Clinicians. Pediatrics. 1999;104: 570-4.

12. CDC. Recommendations regarding the use of vaccines that contain thimerosal as a preservative. MMWR. 1999;48:996-8.

13. Ortega García JA, Ferrís i Tortajada J. Hospitales sostenibles (Parte II). Mercurio: Exposición pediátrica. Efectos adversos en la salud humana y medidas preventivas. Rev Esp Pediatr. 2003;59: 274-91.

14. Clarkson TW, Magos L. The toxicology of mercury and its chemical compounds. Crit Rev Toxicol. 2006;36:609-62.

15. Pichichero ME, Cernichiari E, Lopreiato J, Treanor J. Mercury concentrations and metabolism in infants receiving vacines containing thimerosal: a descriptive study. Lancet. 2002;360:1737-41. 
16. Andrews N, Millar E, Grant A, Stowe J, Osborne $\mathrm{V}$, Taylor $\mathrm{B}$. Thimerosal exposure in infants and developmental disorders: a retrospective cohort study in the United Kingdom does not support a causal association. Pediatrics. 2004;114: 584-91.

17. Ball LK, Ball R, Pratt RD. An Assessment of Thimerosal Use in Childhood Vaccines. Pediatrics. 2001;107;1147-54.

18. OMS. Global Advisory Committee on Vaccine Safety, 20-21 June 2002. Disponible en www. who.int/vaccine_safety/reports/june_2002/en/ index.html [consultado el 01/07/2010].

19. OMS. Vacunas e inmunización: situación mundial. Edición revisada 2003. Ginebra: Organi- zación Mundial de la Salud; 2003. p. 32. Disponible en www.who.int/vaccines-documents/Docs PDF05/Vacunas_e_inmunizacion_completo.pdf [coonsultado el 01/07/2010].

20. DeStefano F. Vaccines and autism: evidence does not support a causal association. Clin Pharmacol Therapeutics. 2007;82:756-9.

21. Hviid A, Stellfeld M, Wohlfahrt J, Melbye M. Association between thimerosal-containing vaccine and autism. JAMA 2003;290:1763-66.

22. Cox NH, Forsyth A. Thimerosal allergy and vacination reactions. Contact Dermatitis. 1988;18: 229-33. 